The Journal of $\mathbf{N}_{\text {onlinear }} \mathbf{S}_{\text {cience and }}$ Applications http://www.tjnsa.com

\title{
A RELATED FIXED POINT THEOREM IN TWO FUZZY METRIC SPACES
}

\author{
ABDELKRIM ALIOUCHE ${ }^{1 *}$, FAYCEL MERGHADI $^{2}$ AND AHCENE DJOUDI ${ }^{3}$ \\ Communicated by D. Mihet
}

\begin{abstract}
We prove a related fixed point theorem for two mappings in two fuzzy metric spaces using an implicit relation which gives fuzzy versions of theorems of [1, 2] and [10].
\end{abstract}

\section{Introduction and preliminaries}

The concept of fuzzy sets was introduced initially by Zadeh [11] in 1965. George and Veeramani [3] modified the concept of fuzzy metric space introduced by [5. Fisher [2], Aliouche and Fisher [1], Telci [10] proved some related fixed point theorems in compact metric spaces. Recently, Rao et.al [7] and [8] proved some related fixed point theorems in sequentially compact fuzzy metric spaces. Motivated by a work due to Popa [6], we have observed that proving fixed point theorems using an implicit relation is a good idea since it covers several contractive conditions rather than one contractive condition.

It is our purpose in this paper to prove fuzzy versions of theorems of [1], [2] and [10].

Definition $1.1([9])$. A binary operation $*:[0,1] \times[0,1] \longrightarrow[0,1]$ is a continuous $t$-norm if it satisfies the following conditions:

(1) $*$ is associative and commutative,

(2) $*$ is continuous,

(3) $a * 1=a$ for all $a \in[0,1]$,

Date: Received: 19 November 2008; Revised: 18 December 2008.

* Corresponding author .

2000 Mathematics Subject Classification. 47H10, 54H25.

Key words and phrases. Fuzzy metric space; implicit relation; sequentially compact fuzzy metric space; related fixed point. 
(4) $a * b \leq c * d$ whenever $a \leq c$ and $b \leq d$, for each $a, b, c, d \in[0,1]$.

Two typical examples of a continuous $t$-norm are $a * b=a b$ and $a * b=$ $\min \{a, b\}$.

Definition $1.2([3])$. The 3-tuple $(X, M, *)$ is called a fuzzy metric space if $X$ is an arbitrary non-empty set, $*$ is a continuous $t$-norm, and $M$ is a fuzzy set on $X^{2} \times[0, \infty)$, satisfying the following conditions for each $x, y, z \in X$ and $t, s>0$,

(FM-1) $M(x, y, t)>0$,

(FM-2) $M(x, y, t)=1$ if and only if $x=y$,

(FM-3) $M(x, y, t)=M(y, x, t)$,

$(\mathrm{FM}-4) M(x, y, t) * M(y, z, s) \leq M(x, z, t+s)$,

(FM-5) $M(x, y,):.(0, \infty) \longrightarrow[0,1]$ is continuous.

Let $(X, M, *)$ be a fuzzy metric space. For $t>0$, the open ball $B(x, r, t)$ with center $x \in X$ and radius $0<r<1$ is defined by

$$
B(x, r, t)=\{y \in X: M(x, y, t)>1-r\} .
$$

A subset $A \subset X$ is called open if for each $x \in A$, there exist $t>0$ and $0<r<1$ such that $B(x, r, t) \subset A$. Let $\tau$ denote the family of all open subsets of $X$. Then $\tau$ is called the topology on $X$ induced by the fuzzy metric $M$. This topology is Hausdorff and first countable.

Example 1.3. Let $X=\mathbb{R}$. Denote $a * b=a . b$ for all $a, b \in[0,1]$. For each $t \in(0, \infty)$, define

for all $x, y \in X$.

$$
M(x, y, t)=\frac{t}{t+|x-y|}
$$

Definition $1.4([3])$. Let $(X, M, *)$ be a fuzzy metric space.

(1) A sequence $\left\{x_{n}\right\}$ in $X$ converges to $x$ if and only if for any $0<\epsilon<1$ and $t>0$, there exists $n_{0} \in \mathbb{N}$ such that for all $n \geq n_{0}, M\left(x_{n}, x, t\right)>1-\epsilon$; i.e., $M\left(x_{n}, x, t\right) \rightarrow 1$ as $n \rightarrow \infty$ for all $t>0$.

(2) A sequence $\left\{x_{n}\right\}$ in $X$ is called a Cauchy sequence if and only if for any $0<\epsilon<1$ and $t>0$, there exists $n_{0} \in \mathbb{N}$ such that for all $n, m \geq n_{0}$, $M\left(x_{n}, x_{m}, t\right)>1-\epsilon$; i.e., $M\left(x_{n}, x_{m}, t\right) \rightarrow 1$ as $n, m \rightarrow \infty$ for all $t>0$.

(3) A fuzzy metric space $(X, M, t)$ in which every Cauchy sequence is convergent is said to be complete.

Lemma $1.5([4])$. For all $x, y \in X, M(x, y,$.$) is a non-decreasing function.$

Definition 1.6. Let $(X, M, *)$ be a fuzzy metric space. $M$ is said to be continuous on $X^{2} \times(0, \infty)$ if

$$
\lim _{n \rightarrow \infty} M\left(x_{n}, y_{n}, t_{n}\right)=M(x, y, t)
$$

whenever $\left\{\left(x_{n}, y_{n}, t_{n}\right)\right\}$ is a sequence in $X^{2} \times(0, \infty)$ which converges to a point $(x, y, t) \in X^{2} \times(0, \infty)$; i.e.,

$$
\lim _{n \rightarrow \infty} M\left(x_{n}, x, t\right)=\lim _{n \rightarrow \infty} M\left(y_{n}, y, t\right)=1 \text { and } \lim _{n \rightarrow \infty} M\left(x, y, t_{n}\right)=M(x, y, t) .
$$

Lemma $1.7([4]) . M$ is a continuous function on $X^{2} \times(0, \infty)$. 
Definition 1.8. $(X, M, *)$ is said to be sequentially compact fuzzy metric space if every sequence in $X$ has a convergent subsequence in it.

Let $\Phi$ be the set of all functions $\phi:[0,1]^{6} \longrightarrow[0,1]$ such that if either $\left(\phi_{a}\right): \phi(u, 1, u, v, v, 1)>0$ or $\left(\phi_{b}\right): \phi(u, u, 1, v, 1, v)>0$ for all $u, v \in(0,1)$, then $u>v$.

Example 1.9. $\phi\left(t_{1}, t_{2}, t_{3}, t_{4}, t_{5}, t_{6}\right)=t_{1}-\varphi\left(t_{2}, t_{3}, t_{4}, t_{5}, t_{6}\right)$, where $\varphi:[0,1]^{5} \rightarrow$ $[0,1]$ which verifies for all $u, v \in(0,1)$,

$\left(\varphi_{a}\right): u>\varphi(1, u, v, v, 1)$ or

$\left(\varphi_{b}\right): u>\varphi(u, 1, v, 1, v)$

implies $u>v$. Then $\phi \in \Phi$.

Example 1.10. Let $\phi\left(t_{1}, t_{2}, t_{3}, t_{4}, t_{5}, t_{6}\right)=t_{1}-\min \left\{t_{2}, t_{3}, t_{4}, t_{5}, t_{6}\right\}$. Then $\phi \in \Phi$.

Example 1.11. $\phi\left(t_{1}, t_{2}, t_{3}, t_{4}, t_{5}, t_{6}\right)=t_{1}-\varphi\left(\min \left\{t_{2}, t_{3}, t_{4}, t_{5}, t_{6}\right\}\right)$, where $\varphi$ : $[0,1] \rightarrow[0,1]$ which satisfies $\varphi(t) \geq t$ for all $t \in[0,1]$. Then $\phi \in \Phi$.

\section{Main results}

Theorem 2.1. Let $\left(X, M_{1}, \theta_{1}\right)$ and $\left(Y, M_{2}, \theta_{2}\right)$ be two fuzzy metric spaces and $T: X \longrightarrow Y, S: Y \longrightarrow X$ be two mappings satisfying

$$
\begin{gathered}
\phi_{1}\left(\begin{array}{c}
M_{1}(S y, S T x, t), M_{1}(x, S y, t), M_{1}(x, S T x, t), \\
M_{2}(y, T x, t), M_{2}(y, T S y, t), M_{2}(T x, T S y, t)
\end{array}\right)>0 \\
\phi_{2}\left(\begin{array}{c}
M_{2}(T x, T S y, t), M_{2}(y, T x, t), M_{2}(y, T S y, t), \\
M_{1}(x, S y, t), M_{1}(x, S T x, t), M_{1}(S y, S T x, t)
\end{array}\right)>0
\end{gathered}
$$

for all $x \in X, y \in Y$ with $x \neq S y, y \neq T x$ and for all $t>0$, where $\phi_{1}, \phi_{2} \in \Phi$. Suppose that one of the following is true:

(a) $\left(X, M_{1}, \theta_{1}\right)$ is sequentially compact and $S T$ is continuous on $X$.

(b) $\left(Y, M_{2}, \theta_{2}\right)$ is sequentially compact and $T S$ is continuous on $Y$.

Then, $S T$ has a unique fixed point $u \in X$ and $T S$ has a unique fixed point $v \in Y$. Further, $T u=v$ and $S v=u$.

Proof. Assume that $\left(X, M_{1}, \theta_{1}\right)$ is sequentially compact and $S T$ is continuous on $X$. Define $\phi: X \rightarrow \mathbb{R}$ by $\phi(x)=M_{1}(x, S T x, t)$ for all $x \in X$ and for every $t>0$. Since $\phi$ a continuous real-valued function on the compact $X$, it attains its maximum; i.e., there exists $u \in X$ such that

$$
\phi(u)=M_{1}(u, S T u, t)=\max \{\phi(x): x \in X\} .
$$

Suppose that $T u \neq T S T u$. Then, $u \neq S T u$.

Putting $y=T u$ and $x=S T u$ in (2.1) we have

$$
\begin{aligned}
& \phi_{1}\left(\begin{array}{c}
M_{1}(S T u, S T S T u, t), M_{1}(S T u, S T u, t), M_{1}(S T u, S T S T u, t), \\
M_{2}(T u, T S T u, t), M_{2}(T u, T S T u, t), M_{2}(T S T u, T S T u, t)
\end{array}\right) \\
= & \phi_{1}\left(\begin{array}{c}
M_{1}(S T u, S T S T u, t), 1, M_{1}(S T u, S T S T u, t), \\
M_{2}(T u, T S T u, t), M_{2}(T u, T S T u, t), 1
\end{array}\right)>0
\end{aligned}
$$


and so by $\left(\phi_{a}\right)$

$$
M_{1}(S T u, S T S T u, t)>M_{2}(T u, T S T u, t) .
$$

Putting $x=u$ and $y=T u$ in (2.2) we get

$$
\begin{aligned}
& \phi_{2}\left(\begin{array}{c}
M_{2}(T u, T S T u, t), M_{2}(T u, T u, t), M_{2}(T u, T S T u, t), \\
M_{1}(u, S T u, t), M_{1}(u, S T u, t), M_{1}(S T u, S T u, t)
\end{array}\right) \\
= & \phi_{2}\left(\begin{array}{c}
M_{2}(T u, T S T u, t), 1, M_{2}(T u, T S T u, t), \\
M_{1}(u, S T u, t), M_{1}(u, S T u, t), 1
\end{array}\right)>0 .
\end{aligned}
$$

Therefore by $\left(\phi_{a}\right)$

$$
M_{2}(T u, T S T u, t)>M_{1}(u, S T u, t) .
$$

From (2.3) and (2.4) we obtain

$$
\begin{aligned}
\phi(S T u) & =M_{1}(\text { STu, STSTu,t }) \\
& >M_{2}(T u, T S T u, t) \\
& >M_{1}(u, S T u, t)=\phi(u)
\end{aligned}
$$

which is a contradiction and so $T S T u=T u$.

Let $T u=v$ and $S v=u$. Then $S T u=S v=u$ and $T S v=T u=v$.

For the uniqueness of $u$, suppose that $S T u^{\prime}=u^{\prime}$ with $u \neq u^{\prime}$. Then, $S T u \neq$ $S T u^{\prime}$ and $T u \neq T u^{\prime}$.

Putting $x=u$ and $y=T u^{\prime}$ in (2.1) we have

$$
\begin{aligned}
& \phi_{1}\left(\begin{array}{c}
M_{1}\left(S T u^{\prime}, S T u, t\right), M_{1}\left(u, S T u^{\prime}, t\right), M_{1}(u, S T u, t), \\
M_{2}\left(T u^{\prime}, T u, t\right), M_{2}\left(T u^{\prime}, T S T u^{\prime}, t\right), M_{2}\left(T u, T S T u^{\prime}, t\right)
\end{array}\right) \\
= & \phi_{1}\left(\begin{array}{c}
M_{1}\left(u, u^{\prime}, t\right), M_{1}\left(u, u^{\prime}, t\right), 1, \\
M_{2}\left(T u, T u^{\prime}, t\right), 1, M_{2}\left(T u, T u^{\prime}, t\right)
\end{array}\right)>0
\end{aligned}
$$

and so by $\left(\phi_{b}\right)$

$$
M_{1}\left(u, u^{\prime}, t\right)>M_{2}\left(T u, T u^{\prime}, t\right) .
$$

Putting $x=u, y=T u^{\prime}$ in (2.2) we get

$$
\begin{aligned}
& \phi_{2}\left(\begin{array}{c}
M_{2}\left(T u, T S T u^{\prime}, t\right), M_{2}\left(T u^{\prime}, T u, t\right), M_{2}\left(T u^{\prime}, T S T u^{\prime}, t\right), \\
M_{1}\left(u, S T u^{\prime}, t\right), M_{1}(u, S T u, t), M_{1}\left(S T u^{\prime}, S T u, t\right)
\end{array}\right) \\
= & \phi_{2}\left(\begin{array}{c}
M_{2}\left(T u, T u^{\prime}, t\right), M_{2}\left(T u, T u^{\prime}, t\right), 1, \\
M_{1}\left(u, u^{\prime}, t\right), 1, M_{1}\left(u, u^{\prime}, t\right)
\end{array}\right)>0 .
\end{aligned}
$$

Therefore by $\left(\phi_{b}\right)$

$$
M_{2}\left(T u, T u^{\prime}, t\right)>M_{1}\left(u, u^{\prime}, t\right) .
$$

Using (2.5) and (2.6) we obtain

$$
M_{1}\left(u, u^{\prime}, t\right)>M_{1}\left(u, u^{\prime}, t\right)
$$

which is a contradiction. Hence, $u$ is the unique fixed point of $S T$. Similarly, we can prove the uniqueness of the fixed points of $T S$. In a similar manner, the theorem holds if $(b)$ is true.

The following example illustrates our Theorem 2.1. 
Example 2.2. Let $\left(M_{1}, X, \theta_{1}\right),\left(M_{2}, Y, \theta_{2}\right)$ be two fuzzy metric spaces such that $M_{1}(x, y, t)=M_{2}(x, y, t)=\frac{t}{t+|x-y|}$ and $X=[3,5], Y=(0,3)$. Define $T:$ $X \rightarrow Y$ and $S: Y \rightarrow X$ by

$$
T x=\left\{\begin{array}{l}
1 \text { if } x \in[3,4[ \\
2 \text { if } x \in[4,5]
\end{array}, S y=\left\{\begin{array}{l}
3 \text { if } y \in(0,1[ \\
4 \text { if } y \in[1,3)
\end{array} .\right.\right.
$$

Let $\phi_{1}=\phi_{2}=\phi$ and $\phi\left(t_{1}, t_{2}, t_{3}, t_{4}, t_{5}, t_{6}\right)=t_{1}-\min \left\{t_{2}, t_{3}, t_{4}, t_{5}, t_{6}\right\}$. We have

$$
S T x=4 \text { for all } x \in[3,5] \text { and } T S y=\left\{\begin{array}{l}
1 \text { if } y \in(0,1[ \\
2 \text { if } y \in[1,3)
\end{array} .\right.
$$

It is easy to see that $\left(X, M_{1}, \theta_{1}\right)$ is sequentially compact, $S T$ is continuous on $X$ and $\left(Y, M_{2}, \theta_{2}\right)$ is not a sequentially compact since $Y$ is not a compact subset of $\mathbb{R}$.

The inequalities (2.1) and (2.2) are satisfied, $S T(4)=4, T S(2)=2, T(4)=2$ and $S(2)=4$.

Taking example 1.9, we get the following corollary which is a fuzzy version of a theorem of [10].

Corollary 2.3. Let $\left(X, M_{1}, \theta_{1}\right)$ and $\left(Y, M_{2}, \theta_{2}\right)$ be two fuzzy metric spaces and $T: X \longrightarrow Y, S: Y \longrightarrow X$ be two mappings satisfying

$$
\begin{aligned}
M_{1}(S y, S T x, t)> & \varphi_{1}\left(M_{1}(x, S y, t), M_{1}(x, S T x, t)\right. \\
& \left.M_{2}(y, T x, t), M_{2}(y, T S y, t), M_{2}(T x, T S y, t)\right) \\
M_{2}(T x, T S y, t)>\quad & \varphi_{2}\left(M_{2}(y, T x, t), M_{2}(y, T S y, t)\right. \\
& \left.M_{1}(x, S y, t), M_{1}(x, S T x, t), M_{1}(S y, S T x, t)\right)
\end{aligned}
$$

for all $x \in X, y \in Y$ with $y \neq T x, x \neq S y$ and for all $t>0$, where $\varphi_{1}$ and $\varphi_{2}$ satisfies $\left(\varphi_{a}\right)$ and $\left(\varphi_{b}\right)$. Suppose that one of the following is true:

(a) $\left(X, M_{1}, \theta_{1}\right)$ is sequentially compact and $S T$ is continuous on $X$.

(b) $\left(Y, M_{2}, \theta_{2}\right)$ is sequentially compact and $T S$ is continuous on $Y$.

Then, $S T$ has a unique fixed point $u \in X$ and $T S$ has a unique fixed point $v \in Y$. Further, $T u=v$ and $S v=u$.

Taking example 1.10, we get the following corollary which is a fuzzy version of a theorem of [2].

Example 2.4. Let $\left(X, M_{1}, \theta_{1}\right)$ and $\left(Y, M_{2}, \theta_{2}\right)$ be two fuzzy metric spaces and $T: X \longrightarrow Y, S: Y \longrightarrow X$ be two mappings satisfying

$$
\begin{aligned}
M_{1}(S y, S T x, t)> & \min \left\{M_{1}(x, S y, t), M_{1}(x, S T x, t),\right. \\
& \left.M_{2}(y, T x, t), M_{2}(y, T S y, t), M_{2}(T x, T S y, t)\right\} \\
M_{2}(T x, T S y, t)> & \min \left\{\left(M_{2}(y, T x, t), M_{2}(y, T S y, t)\right.\right. \\
& \left.M_{1}(x, S y, t), M_{1}(x, S T x, t), M_{1}(S y, S T x, t)\right\}
\end{aligned}
$$

for all $x \in X, y \in Y$ with $x \neq S y, y \neq T x$ and for all $t>0$. 
Suppose that one of the following is true:

(a) $\left(X, M_{1}, \theta_{1}\right)$ is sequentially compact and $S T$ is continuous on $X$.

(b) $\left(Y, M_{2}, \theta_{2}\right)$ is sequentially compact and $T S$ is continuous on $Y$.

Then, $S T$ has a unique fixed point $u \in X$ and $T S$ has a unique fixed point $v \in Y$. Further, $T u=v$ and $S v=u$.

Acknowledgements. The authors would like to thank very much Dr. D.

Mihet for his valuable comments and suggestions.

\section{REFERENCES}

[1] A. Aliouche and B. Fisher, Fixed point theorems for mappings satisfying implicit relation on two complete and compact metric spaces, Applied Mathematics and Mechanics, 27 (9) (2006), 1217-1222. (document) 1

[2] B. Fisher, Fixed point on two metric spaces, Glasnik Mat., 16 (36) (1981), 333-337. (document), 1. 2

[3] A. George and P. Veeramani, On some result in fuzzy metric space, Fuzzy Sets Syst., 64 (1994), 395-399. 1, $1.2,1.4$

[4] M. Grabiec, Fixed points in fuzzy metric spaces, Fuzzy Sets Syst., 27 (1988), 385-389. 1.5 . 1.7

[5] I. Kramosil and J. Michalek, Fuzzy metric and statistical metric spaces, Kybernetica, 11 (1975), 326-334. 1

[6] V. Popa, Some fixed point theorems for compatible mappings satisfying an implicit relation, Demonstratio Math., 32 (1999),157-163. 1

[7] K. P. R. Rao, N. Srinivasa Rao T. Ranga Rao and J. Rajendra Prasad, Fixed and related fixed point theorems in sequentially compact fuzzy metric spaces, Int. Journal of Math. Analysis, Vol. 2, 2008, no. 28, 1353-1359 1

[8] K. P. R. Rao, Abdelkrim Aliouche and G. Ravi Babu, Related Fixed Point Theorems in Fuzzy Metric Spaces, The Journal of Nonlinear Sciences and its Application, 1 (3) (2008), 194-202. 1

[9] B. Schweizer and A. Sklar, Statistical metric spaces. Pacific J. Math., 10 (1960), 313-334. 1.1

[10] M. Telci, Fixed points on two complete and compact metric spaces, Applied Mathematics and Mechanics, 22 (5) (2001), 564-568. (document), 1 , 2

[11] L. A. Zadeh, Fuzzy sets, Inform and Control, 8 (1965), 338-353. 1

${ }^{1}$ Department of Mathematics, University of Larbi Ben M' Hidi, Oum-El-Bouaghi, 04000, Algeria.

E-mail address: alioumath@yahoo.fr

${ }^{2}$ Department of Mathematics, University of Tebessa, 12000, Algeria.

E-mail address: faycel_mr@yahoo.fr

${ }^{3}$ Université de Annaba, Faculté des sciences, Département de mathématiques, B. P. 12, 23000, Annaba, AlgÉrie

E-mail address: adjoudi@yahoo.com 